\title{
PERFIL NUTRICIONAL DOS PACIENTES COLOSTOMIZADOS DE UM HOSPITAL PÚBLICO DO INTERIOR PAULISTA
}

Luis Henrique Alves de Lima', Sílvia Coutinho Pain', Francielle Ferreira Serrano', Monique de Caldas Raimundo', Sandra Cristina Genaro', Sabrina Alves Lenquiste', Gabrielle Gomes dos Santos Ribeiro', Fabíola de Azevedo Mello², Marcela de Andrade Bernal Fagiani ${ }^{1}$

${ }^{1}$ Universidade do Oeste Paulista - UNOESTE, Curso de Nutrição.c ${ }^{2}$ Universidade do Oesta Paulista - UNOESTE, Doutorado em Fisiopatologia e Saúde Animal. .e-mail: marcelafagiani16@gmail.com

\section{RESUMO}

A colostomia é um procedimento cirúrgico, que pode tornar-se necessário devido a alguns fatores, como câncer colorretal, doenças inflamatórias intestinais e traumas. Exige cuidados especiais, com relação ao estado nutricional, hábito intestinal, consistência das fezes, monitoramento do orifício e sua higienização, a fim de evitar possíveis complicações. O objetivo do presente estudo foi avaliar o perfil nutricional de indivíduos em uso da bolsa de colostomia. O estudo foi exploratório descritivo transversal, com abordagem quantitativa. Foram entrevistados 20 indivíduos de um ambulatório de estomaterapia em um hospital público do interior paulista, por meio da aplicação de questionário e realização da avaliação nutricional. A maior parcela da população apresentou eutrofia pela classificação do índice de massa corporal, e depleção por meio dos resultados da adequação de circunferência muscular do braço e área muscular do braço. A principal causa para o uso da bolsa de colostomia foi o câncer colorretal.

Palavras-chave: Colostomia, desnutrição, ostomia, estoma cirúrgico.

\section{NUTRITIONAL PROFILE OF THE COLOSTOMIZED PATIENTS OF A PUBLIC HOSPITAL IN THE INTERIOR OF SÃO PAULO}

\begin{abstract}
Colostomy, is a surgical procedure, which may become necessary, because of some factors, such as colorectal cancer, inflammatory bowel diseases and trauma. Requires special care, regarding the nutritional status, bowel habit, stool consistency, orifice monitoring and hygiene, in order to prevent complications. The aim of study, was evaluate the nutritional profile of individuals using the colostomy bag. Were interviewed twenty individuals from a stomatherapy ambulatory, in a public hospital in the interior of São Paulo. This study has a descriptive and exploratory charater, with a quantitative approach, through the application of a questionnaire and the accomplishment of the nutritional evaluation. The largest part of the population, presented eutrophy due to body mass index classification, and depletion through the results of the adequacy of arm circumference and arm muscle area. The main cause for the use of the colostomy bag, was colorrectal cancer.
\end{abstract}

Keywords: Colostomy, mal nutrition, ostomy, surgical stomas.

\section{INTRODUÇÃO}

A colostomia é um procedimento cirúrgico, que surgiu no final do século XIX como uma alternativa ao tratamento de doenças de cólon $^{1,2}$, na qual é realizada uma abertura na parede abdominal (estoma), podendo ser temporária ou permanente, e nela é ligada uma terminação do intestino, pela qual as fezes e gases passarão a ser eliminados, possuindo uma bolsa adesiva coletora dos produtos intestinais junto ao estoma ${ }^{3}$. 
Existem alguns fatores que podem levar o indivíduo a necessitar de ostomia, tais como doença de Chagas, doença de Chron, inflamações, corpos estranhos introduzidos no reto, amputação do reto, fístulas retovaginais, perfurações cólicas, lesões extensas ao redor do ânus, neoplasias e acidentes que causem impactos na região abdominal, ressaltando que o local da ostomia, depende da região intestinal acometida ${ }^{4,5}$. A neoplasia de cólon pode exigir a realização de ostomia, sendo responsável, em média, por $83 \%$ dos casos de ostomias nestes indivíduos ${ }^{6}$.

A colostomia requer cuidados especiais, de preferência, prestados por profissionais experientes em lidar com indivíduos ostomizados, principalmente no momento da higienização dos curativos e da bolsa coletora, controle das eliminações que inclui fatores associados à alimentação, como consistência das fezes, odor, e frequência intestinal, além de monitoramento do orifício e das possíveis reações ou complicações ${ }^{7}$, que podem causar desconfortos ou até mesmo constrangimentos aos indivíduos estomizados ${ }^{8}$.

Os ostomizados sofrem prejuízos no seu estado nutricional, que podem estar relacionados ao não fracionamento das refeições diárias, devido ao pouco conhecimento da influência da alimentação sobre a colostomia ${ }^{9}$.

No dia 16 de novembro de 2009, em homenagem ao dia nacional dos estomizados, foi publicada no diário oficial da união, a portaria do Ministério da Saúde, número 400, que estabelece as diretrizes nacionais para atenção à saúde de indivíduos ostomizadas no âmbito do Sistema Único de Saúde (SUS), destacando a atuação e importância do nutricionista para estes indivíduos ${ }^{10}$.

A dieta completa e equilibrada, orientada por um nutricionista, é um elemento imprescindível para evitar a desnutrição e promover a adequação do consumo de nutrientes específicos, o que evidencia a importância da nutrição adequada destes indivíduos ${ }^{11}$.

A assistência nutricional deve se iniciar assim que o diagnóstico favorável à colostomia é feito, visto que para realizar a colostomia, é necessário submeter o indivíduo a uma cirurgia radical o que pode levar a perda de peso e no pós-cirúrgico, as alterações alimentares podem causar alterações no equilíbrio hidroeletrolítico do organismo ${ }^{5}$.
Depois da necessidade de uma colostomia, muitos pacientes são capazes de ter uma dieta normal, porém, estudos apontam para a necessidade de realizar educação nutricional nesta população, com o intuito de orientar os pacientes sobre como lidar com as mudanças nos hábitos alimentares e identificar quais alimentos deve-se preferir ou evitar ${ }^{12}$.

Sendo assim, o objetivo do presente estudo foi identificar o perfil nutricional de pacientes com uso da bolsa de colostomia.

\section{METODOLOGIA}

O estudo foi exploratório descritivo transversal, com abordagem quantitativa. Ao todo, foram abordados 20 indivíduos atendidos num ambulatório para consulta de estomaterapia num hospital público. Os indivíduos que aceitaram participar da pesquisa assinaram um Termo de Consentimento Livre e Esclarecido (TCLE), contendo informações sobre os objetivos do trabalho e os procedimentos aos quais seriam submetidos. O projeto obedeceu aos critérios da Ética em Pesquisa com Seres Humanos, conforme a Resolução no 466/2012, do Conselho Nacional de Saúde (CNS) e foi aprovado pelo Comitê de Ética e Pesquisa (CEP) da Universidade do Oeste Paulista (Unoeste; CAAE: 83149718.3.0000.5515).

Para a coleta de dados, os pacientes foram abordados no ambulatório de um hospital público em seu retorno ao local a cada quatro meses, por meio de agendamento. Aqueles que estiveram em retorno no período do estudo, no momento da consulta de estomaterapia, foram convidados para participar deste trabalho, independente do tempo em que estivessem no período pós-cirúrgico.

Dessa forma, a população alvo do estudo foi escolhida por conveniência, de forma aleatória e não probabilística. Tratou-se de um estudo de campo, quantitativo, qualitativo, transversal e prospectivo. Foram excluídos indivíduos confusos, que não conseguissem ser submetidos à avaliação nutricional ou responder às perguntas da entrevista do estudo e àqueles que não possuíam idade mínima de 18 anos.

Os indivíduos foram submetidos a um questionário não-padronizado, contendo perguntas relacionadas aos aspectos sociodemográficos, clínicos (motivo da colostomia, há quanto tempo possui colostomia, temporalidade do estoma e comorbidades), e se já havia realizado acompanhamento com um nutricionista. 
Em seguida, foi realizada a avaliação antropométrica. Para a aferição do peso, os participantes foram posicionados em pé, sem calçados, com roupas leves e braços ao longo do corpo, em uma balança digital com capacidade para $150 \mathrm{~kg}$ (G-Tech ${ }^{\circ}$, Accumed-Glicomed, Brasil).

Para a medida da altura, realizou-se uma adaptação de acordo com o Instituto Brasileiro de Geografia e Estatística (IBGE) ${ }^{13}$. Dessa forma, utilizou-se fita métrica de $200 \mathrm{~cm}$, fixada na parede sem rodapé, onde os indivíduos foram posicionados em pé, com o corpo ereto, braços ao longo do corpo, calcanhares encostados na parede, com o auxílio de uma régua, foi realizada a leitura da altura.

Por meio dos resultados do peso e altura, foi calculado o índice de massa corporal (IMC), obtido pelo resultado da razão entre o peso corporal em quilogramas e a altura em metros elevada ao quadrado $\left(I M C=\text { peso/altura }{ }^{2}\right)^{14}$ e foi interpretado de acordo com os valores de referência ${ }^{15}$.

Em seguida, para avaliar as reservas energéticas e musculares dos pacientes, foram realizadas respectivamente a dobra cutânea tricipital (DCT) (mm), utilizando adipômetro com escala de 0 a $60 \mathrm{~mm}$, com mola de precisão constante de $10 \mathrm{~g} / \mathrm{mm}^{2}$ (Lange ${ }^{\circledR}$, TBW, Estados Unidos da América), e realizou-se três vezes a aferição, sendo considerada e utilizada, a mediana entre os valores encontrados.

Seguidamente, foi medida a circunferência do braço ( $C B$; em $\mathrm{cm}$ ), utilizando fita métrica flexível graduada de 0 a $150 \mathrm{~cm}$, posicionada no ponto médio entre o processo acromial e o olécrano da ulna, circundando o braço. As medidas de DCT e CB, foram realizadas no lado não dominante dos indivíduos ${ }^{16}$. Com os valores encontrados da CB e DCT, foi realizado o cálculo da circunferência muscular do braço $(\mathrm{CMB})$, onde $\mathrm{CMB}(\mathrm{cm})=\mathrm{CB}(\mathrm{cm})-[0,314 \mathrm{x}$ $\mathrm{DCT}]^{17}$. Foram realizados os cálculos das porcentagens de adequações da $\mathrm{CB}, \mathrm{DCT}, \mathrm{CMB}$ e área muscular do braço corrigida $(\mathrm{AMBC})^{18}$.

Após a coleta de dados, todas as informações foram submetidas à análise estatística, por meio do Software estatístico Action Stat ${ }^{\oplus}$ (Portal Action, Campinas, Brasil). Foi realizada a análise descritiva das variáveis, com os cálculos de frequências e medidas numéricas como média, desvio-padrão, coeficiente de variação e foram construídos os gráficos. Posteriormente, realizou-se a análise de correlação de Pearson, a fim de verificar a existência de associação entre alguns pares de variáveis.

\section{RESULTADOS}

O estudo foi realizado com 20 indivíduos com idade superior a 18 anos, sendo que metade possuía entre 46 e 60 anos e a outra metade, idade superior a 60 anos. $50 \%$ dos indivíduos eram do sexo feminino e do sexo masculino, respectivamente.

A principal causa relacionada ao uso da bolsa de colostomia foi o câncer colorretal (71\%; Tabela 1).

Tabela 1. Causas para o uso da bolsa de colostomia.

\begin{tabular}{lc}
\hline Motivo & $\%$ \\
\hline Câncer colorretal & 71 \\
Diverticulite & 14 \\
Infecção na camada mucosa & 5 \\
Volvo intestinal & 5 \\
Doenças inflamatórias intestinais & 5 \\
\hline
\end{tabular}

Quando questionados se buscaram um nutricionista para orientações sobre a alimentação com relação ao uso da bolsa de colostomia e acompanhamento nutricional, $58 \%$ relataram que não haviam procurado.

Segundo alguns resultados da avaliação nutricional, os indivíduos apresentaram eutrofia pelo IMC em sua maioria $(n=11)$. Ao avaliar as reservas musculares, a maioria dos indivíduos apresentou uma depleção grave $(n=9)$ pelo resultado do cálculo da $\mathrm{CMB}$ e de sua adequação e pelo cálculo da $\mathrm{AMBc}$.

Ao comparar a situação nutricional entre os sexos por meio de proporções, verificou-se que as mulheres apresentaram maior percentual de eutrofia pelo IMC (38\%) do que os homens (20\%). Os homens apresentaram maior percentual de eutrofia (20\%) do que as mulheres (15\%) de acordo com a CMB. Para os resultados da $\mathrm{AMBc}$, houve predominâcia de depleção grave para homens $(25 \%)$ e mulheres $(20 \%)$, mas ainda assim, os homens tiveram uma maior depleção muscular.

Na Tabela 2 estão demonstradas as correlações por meio do teste de Pearson entre o tempo de uso de colostomia com o estado nutricional, demonstrado por meio do IMC. Podese dessa forma interpretar que quanto maior o 
tempo do uso da bolsa, maiores podem ser os

índices de desnutrição.

Tabela 2. Correlação do estado nutricional com o gênero, tempo de uso da bolsa de colostomia e faixa etária.

\begin{tabular}{l|c|c|c}
\hline & \multicolumn{3}{|c}{ Estado Nutricional } \\
\hline Variáveis & IMC & $\begin{array}{c}\text { Adequação da } \\
\text { CMB (\%) }\end{array}$ & AMBc (cm $\mathbf{c m}^{\mathbf{2}}$ \\
\hline Sexo & 0,1062 & 0,9999 & 0,4885 \\
Tempo de uso da bolsa & $0,0411^{*}$ & 0,3346 & 0,4946 \\
Faixa etária & 0,1412 & 0,6075 & 0,9999
\end{tabular}

* P $<0,05$. IMC - índice de massa corpórea; CMB - circunferência muscular do braço; $\mathrm{AMBC}$ - área muscular do braço corrigida.

Os indivíduos que utilizam a bolsa de colostomia há mais de um ano, apresentaram em sua maioria, depleção muscular grave segundo os dados da AMBc (25\%) e depleção leve pelo cálculo da adequação da CMB (25\%).

Os participantes com idade superior a 60 anos apresentaram eutrofia pelo cálculo da AMBC em maior proporção $(25 \%)$ do que os indivíduos na faixa etária de 46 a 60 anos (20\%). E pelo cálculo da adequação da $\mathrm{CMB}$, indivíduos com idade superior a 60 anos tiveram em sua maioria, depleção leve (25\%). De acordo com o IMC, a maioria dos indivíduos com idade superior a 60 anos apresentaram eutrofia (30\%).

\section{DISCUSSÃO}

Diante da prevalência de indivíduos que relataram o câncer colorretal como a principal causa da colostomia, este estudo corroborou com o estudo de Salomé e Almeida ${ }^{19}$, no qual as neoplasias apresentaram significância $(p<0,003)$ dentre as causas de colostomia, considerando valor de $p<0,05$, e com o estudo de Aguiar et al. ${ }^{20}$, em que 54 indivíduos de 117 entrevistados, apresentaram o câncer colorretal como a causa mais frequente para o uso da bolsa de colostomia.

A doença ou condição que levou ao uso da bolsa de colostomia pode determinar por quanto tempo o indivíduo utilizará este dispositivo. Dentre essas causas, pode-se citar o câncer, abdome agudo $^{20}$, diverticulite e volvo intestinal. Diante da amostra estudada, verificouse que $50 \%$ dos entrevistados possuíam a bolsa por um período superior a 12 meses.

A perda de peso é um fator que influencia na qualidade de vida dos colostomizados, pois segundo Selau et al. $^{21}$, essa é uma condição comum nessa população, e isso pode ocorrer mediante as mudanças alimentares sem orientação adequada. Os pacientes muitas vezes restringem sua alimentação por conta própria, e dessa forma, podem ficar desidratados e de certa forma, suas reservas de vitaminas e minerais podem ficar reduzidas.

Sendo assim, a procura pelo nutricionista pode trazer maior conforto ao colostomizado, pois terá conhecimento sobre quais escolhas alimentares são melhor para sua saúde, visto que este profissional auxiliará na adequação da alimentação e da ingestão dietética ${ }^{22}$. Porém, no presente estudo, a maioria dos entrevistados (58\%), não haviam se consultado com um nutricionista.

Attolini e Gallon ${ }^{23}$ relataram que o IMC também é um preditor de qualidade de vida nessa população, mas esse índice deve ser avaliado em conjunto com outras informações nutricionais. Dos 20 indivíduos entrevistados, 11 foram classificados como eutróficos pelo IMC, dessa forma, deve-se considerar as demais medidas antropométricas para classificar a situação nutricional, visto que os indivíduos também apresentaram depleção muscular pela $A M B c$ e CMB, apesar de estarem eutróficos pelo IMC.

Apesar de o IMC ser um tipo de classificação do estado nutricional, segundo Ribeiro et al. ${ }^{24}$, este método deve estar associado à outros métodos, para garantir a fidedignidade da classificação do estado nutricional, pois o mesmo, pode mascarar a presença de desnutrição, classificando um indivíduo como eutrófico quando na verdade, este indivíduo não está em estado de eutrofia. 
Para avaliar o estado nutricional, foi realizado o cálculo da AMB, que segundo Souza ${ }^{25}$, a $A M B$ deve ser estimada por meio das medidas da CB e DCT, a fim de expressar a reserva muscular, que se associa à presença de desnutrição protéico-calórica, podendo repercutir em uma piora do estado nutricional e consequentemente, causa uma piora clínica aos pacientes, principalmente em hospitalizados.

Além da $A M B$, foi realizado o cálculo da $\mathrm{CMB}$, que segundo Ganen et al. $^{26}$, pode ser considerado uma medida indicativa de reserva de tecido muscular, sem a realização da correção da área óssea.

Foi possível concluir que a principal causa do uso da bolsa de colostomia foi o câncer colorretal. A população estudada apresentou desnutrição protéica de acordo com os cálculos realizados por meio da avaliação antropométrica. A maioria dos indivíduos não procurou por um nutricionista desde o momento em que necessitou utilizar a bolsa de colostomia.

\section{DECLARAÇÃO DE CONFLITOS DE INTERESSE}

Os autores declaram não haver qualquer potencial conflito de interesse que possa interferir na imparcialidade deste trabalho científico.

\section{REFERÊNCIAS}

1. Sonobe HM, Barichello E, Zago MMF. A visão do colostomizado sobre o uso da bolsa de colostomia. Rev Bras Cancerol. 2020;48(3):341-8.

2. Fonseca $A Z$, Uramoto $E$, Santos-Rosa $O M$, Santin S, Ribeiro-JR M. Fechamento de colostomia: fatores de risco para complicações. Arq Bras Cir Dig. 2015;30(4):231-4. DOI: https://doi.org/10.1590/0102-

$\underline{6720201700040001}$

3. Lopes GF. Análise do conhecimento de enfermagem a pacientes com ostomia intestinal. [Monografia]. Especialização em Linhas de Cuidado em Enfermagem, Departamento de Enfermagem. Universidade Federal de Santa Catarina, Florianópolis, 2014.

4. Barbutti RCS, Silva MCP, Abreu MAL. Ostomia, uma difícil adaptação. Rev SBPH. 2008;11(2):2739.

5. Silva DG, Bezerra ALQ, Siqueira KM, Paranaguá TTB, Barbosa MA. Influência dos hábitos alimentares na reinserção social de um grupo de estomizados. Rev Eletr Enf. 2010;12(1):56-62. DOI: https://doi.org/10.5216/ree.v12i1.5246

6. Stumm EMF, Oliveira ERM, Kirchner RM. Perfil de pacientes ostomizados. Sci Med. 2008;18(1):26-30.

7. Santana JCB, Souza AB, Dutra BS. Perceptions of a group of nurses on the process of taking care of patients with permanent colostomy. Rev Enferm UFPE. 2011;5(7):1710-5. DOI: https://doi.org/10.5205/reuol.1262-12560-1LE.0507201119

8. Oliveira AL. Qualidade de vida relacionada à saúde e perfil nutricional de portadores de derivação intestinal - colostomia e íleostomia. [Dissertação]. Juiz de Fora: Universidade Federal de Juiz de Fora, 2017.

9. Barros LS. Elaboração de cartilha informativa e propostas de cardápio, com evolução de consistência para pacientes ostomizados do hospital universitário de Brasília. [Monografia]. Brasília: Universidade Federal de Brasília. Brasília, 2014.

10. Brasil. Ministério da Saúde. Portaria n. 400, de 16 de novembro de 2009. Estabelece as diretrizes nacionais para a atenção à saúde das pessoas ostomizadas no âmbito do Sistema Único de Saúde - SUS, a serem observadas em todas as unidades federadas, respeitadas as competências das três esferas de gestão. Diário Oficial da União, Brasília, DF, 16 nov. 2009. Seção 1, p.41.

11. Palludo KF, Silveira DA, Vanz R, Petuco VM. Avaliação da dieta de pacientes com colostomia definitiva por câncer colorretal. Rev Estima. 2016;9(1):1-8.

12. Mahan LK, Escott-Stump S, Raymond JL. Krause: alimentos, nutrição e dietoterapia. Rio de Janeiro: Editora Elselvier; 2015.

13. Instituto Brasileiro de Geografia e Estatística (IBGE). Pesquisa Nacional de Saúde 2013. Manual de Antropometria. Rio de Janeiro, RJ. 2013.

14. Ribeiro SML, Melo CM, Tirapegui J. Avaliação nutricional teoria e prática. In: Avaliação da composição corporal por técnicas 
antropométricas. Rio de janeiro: GuanabaraKoogan; 2018. p.43-7.

15. ABESO. Diretrizes Brasileiras de Obesidade. São Paulo, SP. 2016.

16. Machado RSP, Coelho MASC, Coelho KSC. Percentual de gordura corporal em idosos: comparação entre os métodos de estimativa pela área adiposa do braço, pela dobra cutânea tricipital e por bioimpedância tetrapolar. Rev Bras Geriatr Gerontol. 2012;12(2):201-21. DOI: https://doi.org/10.1590/S1809$\underline{98232010000100003}$

17. Araújo GT. Antropometria. Associação Brasileira de Nutrologia. Curso Nacional de Nutrologia. 2015. 13.f.

18. Melo APF, Salles RK, Vieira FGK, Ferreira MG. Métodos de estimativa de peso corporal e altura em indivíduos hospitalizados: uma análise comparativa. RBCDH. 2014;16(4):475-84. DOI: https://doi.org/10.5007/1980$\underline{0037.2014 v 16 n 4 p 475}$

19. Salome GM, Almeida SA. Association of sociodemographic and clinical factors with the self-image and self-esteem of individuals with intestinal stoma. J. Coloproctol. 2014;34(3):15966.

DOI:

https://doi.org/10.1016/i.jcol.2014.05.005

20. Aguiar JC, Pereira APS, Galisteu KJ, Lourenção LG, Pinto $\mathrm{MH}$. Aspectos sociodemográficos e clínicos de estomizados intestinais provisórios. Rev Min Enferm. 2017;21-e-1013. DOI: https://doi.org/10.5935/1415-2762.20170023

21. Selau CM, Limberger LB, Silva MEN, Oliveira FS, Margutti KMM. Percepção dos pacientes com estomia intestinal em relação às mudanças nutricionais e estilo de vida. Texto Contexto Enferm. 2019;18:e20180156. DOI: https://doi.org/10.1590/1980-265x-tce-2018-

$\underline{0156}$

22. Gomes RM, Triani FS, Silva CAF. Conhecimento nutricional de praticantes de treinamento de força. RBNE. 2017;11(65):610-7.

23. Attolini RC, Gallon CW. Qualidade de vida e perfil nutricional de pacientes com câncer colorretal colostomizados. Rev Bras Coloproct.
2010;30(3):289-98.

DOI:

https://doi.org/10.1590/S0101-

$\underline{98802010000300004}$

24. Ribeiro MMC, Araújo ML, Cunha LM, Ribeiro DMC, Pena GG. Análise de diferentes métodos de avaliação do estado nutricional de pacientes em hemodiálise. Rev Cuid. 2014;6(1):932-40. DOI: https://doi.org/10.15649/cuidarte.v6i1.163

25. Souza A. Ângulo de fase e área muscular do braço: indicadores de mortalidade em pacientes submetidos ao transplante de células-tronco hematopoíeticas alogênico. [Dissertação]. Curitiba: Universidade Federal do Paraná, 2016.

26. Ganen AP, Lira AG, Blachiunas RE. Avaliação nutricional teoria e prática. In: Avaliação nutricional de adolescentes. Rio de Janeiro: Guanabara-Koogan; 2018. p.226. 